\title{
Análise do perfil de automedicação em mulheres idosas brasileiras
}

\author{
Analysis of the self-medication pattern \\ among Brazilian elderly women
}

\author{
Paula Chagas Bortolon ${ }^{1}$ \\ Eloá Fátima Ferreira de $M$ edeiros ${ }^{1}$ \\ Janeth Oliveira Silva $\mathrm{N}$ aves ${ }^{1,2}$ \\ M argô Gomes deO liveira Karnikowski ${ }^{1,3}$ \\ Otávio deTolêdo Nóbrega ${ }^{1,3}$
}

${ }^{1}$ Curso de Farmácia, Universidade Católica de Brasília. EPCT QS 7/lote 1, Águas Claras. 72030-170 Brasília DF. nobrega@pq.cnpq.br ${ }^{2}$ Curso de Ciências Farmacêuticas, Faculdade deCiências da Saúde, Universidade de Brasília. ${ }^{3}$ Programa de

Pós-Graduação em Gerontologia, Universidade Católica de Brasília.
Abstract The elderly consume more medications than other age groups, being susceptible to irrational use of therapeutic drugs. This work describes a cross-sectional study that evaluated selfmedication practiced by elderly women, who were assisted at the Elderly Care Facility of the hospital of the Catholic University of Brasilia. The socio-economical variables analyzed were age group, educational level and monthly family income. The pharmaceuticals were evaluated according to prevalence, suitability for the age group and possible drug interactions. Among the 218 patients interviewed, $26 \%$ declared self-medication practices, part of them (40\%) were prescription drugs. Anti-inflammatory, analgesic and antipyretic drugs were the most frequently consumed without professional orientation, followed by phytotherapeutic/natural and cardiovascular agents. Among the self-medicated patients $65 \%$ had incomplete or no formal basic education whereas $32 \%$ had earnings equal to or inferior to one minimum wage. The mean consumption of drugs by self-medication was not influenced by socio-economical variables. The practice of selfmedication involves potentially harmful drug interactions and use of agents inappropriate for the age group.

Key words Pharmacotherapy, Self-medication, Elderly, Drug interactions, Drug-related problem
Resumo Idosos compõem o grupo etário mais medicalizado na sociedade, o que podelevar ao uso irracional defármacos. Estetrabalho descreveestudo transversal que avaliou a automedicação de idosas atendidas no ambulatório de Atenção ao I doso do Hospital da Universidade Católica de Brasília. As variáveis socioeconômicas analisadas consistiram na faixa etária, escolaridade e renda familiar mensal. As classes medicamentosas envolvidas em automedicação foram analisadas conforme prevalência, adequação à faixa etária e possíveis interações medicamentosas. Das 218 pacientes entrevistadas, $26 \%$ relataram praticar automedicação. Parcela (40\%) desses medicamentos era de venda sob prescrição. M edicamentos antiinflamatórios, anal gési cos e antipi réticos foram os mais usados sem orientação profissional, seguido pelos fitoterápicos/medicamentos naturais e cardiovasculares. Entre as pacientes automedicadas, $65 \%$ apresentaram baixa escolaridade, enquanto $32 \%$ apresentaram renda menor ou igual a um salário mínimo. 0 consumo por automedicação não se mostrou variar conforme a condição socioeconômica. Foram observadas interações medicamentosas potencialmente severas e uso impróprio envolvendo os eventos de automedicação. Palavras-chave Farmacoterapia, Automedicação, I doso, Interação medicamentosa, Problema relacionado a medicamentos 
Introdução

A população de idosos brasileiros vem crescendo muito como conseqüência do aumento da expectativa de vida, sendo considerado um reflexo das ações de saúde pública e avanços médicotecnológicos implementados a partir de $1940^{1}$. Quando comparada com o universo masculino, a expectativa de vida das mulheres vem sendo apontada como maior ${ }^{2,3}$.

Em menos de quarenta anos, o Brasil migrou deum perfil de mortalidadetípico de uma população jovem para um quadro caracterizado por enfermidades crônicas emúltiplas, sobretudo nas faixas etárias mais avançadas ${ }^{2,4}$. A abordagem médica tradicional, focada em uma queixa principal, ea conduta médica de reunir as queixaseos sinais em um único diagnóstico não são adequados ao idoso $0^{4}$. Idosos podem apresentar comprometimento de mais de um órgão ou sistema, o que os faz candidatos a acompanhamento mé dico constanteeà polifarmacoterapia. Desta prática, podem decorrer sinergismoseantagonismos não desejados, descumprimento das prescrições dos produtos essenciais na clínica e gastos excessivos com os de uso desnecessário ${ }^{5}$.

Os idosos são provavelmente o grupo mais exposto à polifarmacoterapia na sociedade ${ }^{6}$. A média de medicamentos utilizados por estes indivíduos é de dois a cinco medicamentos $5^{5,7}$. Em relação aos idosos residentes em instituições geriátricas, esse número pode ser maior que sete medicamentos por paciente ${ }^{8,9}$. Alguns estudos têm demonstrado que as mulheres, em relação à idade, formam o grupo social que mais utiliza medicamentos ${ }^{10}$, provavel mente por conseqüência de um pior estado funcional e de saúde autoreferidos eevidenciado por maior número desintomas de depressão e hospitalizações ${ }^{11,12}$.

Estima-se que 30\% das admissões hospitalares de pacientes idosos são relacionadas a problemas com medicamentos, incluindo efeitos tóxi$\cos$ advindos do seu uso ${ }^{13}$. Problemas relacionados a medicamentos (PRMs) são entendidos como problemas de saúde relacionados à farmacoterapia, podendo ter origem no sistema de saúde, em fatores biopsicossociais, no atendimento prestado por profissionais de saúde e na utilização de medicamentos, interferindo nos resultados terapêuticos e na qualidade de vida do usuário ${ }^{14}$. Na sociedade norte-americana, PRM s foram responsáveis por $5 \%$ a $15 \%$ dos casos de hospitalização de idosos em 1988, e por aproximadamente $45 \%$ dos casos de readmissão hospitalar em 1991. Segundo Perry ${ }^{15}$, os PRM s foram associados a uma mortalidade anual de $106 \mathrm{mil}$ indivíduos, com um custo de US\$ 85 bilhões. No Brasil, o impacto dos problemas com medicamentos sobre as internações de idosos permanece por ser determinado. Os estudos disponíveis sobre o sistema oficial de informações hospitalares, como aquele realizado por Loyola-Filho ecolaboradores ${ }^{16}$, deixam de explorar possíveis motivos medicamentosos como causa para as internações observadas. Os únicos dados brasileiros dão conta de que medicamentos são responsáveis por $28 \%$ dos casos de intoxicação humana no país ${ }^{17}$ e por $6,6 \%$ do total de admissões hospitalares ${ }^{18}$, sem quehaja avaliação específica do segmento idoso. Muitos desses eventos constituem problemas previsíveis em pacientes idosos, sobretudo a ocorrência de depressão, confusão e constipação, além dos casos de imobilidade e quedas por decorrência de fraturas ósseas relacionados ao uso de determinadas medicações ${ }^{13,19}$.

Como fator de risco para os PRMs, encontra-se a automedicação, que é o uso de medicamento sem a prescrição, orientação e ou acompanhamento do médico ou dentista ${ }^{20}$. A familiaridade do leigo com os medicamentos, as experiências positivas anteriores ea dificuldade deacesso a serviços de saúde são fatores que contribuem para a automedicação ${ }^{21}$. A pesar de constituir prática que pode tornar fácil o autocuidado, permitir a recuperação de pequenas indisposições e aliviar a sobrecarga dos serviços médicos ${ }^{22}$, existe a possibilidade de agravamento de problemas de saúde como, por exemplo, os causados por doenças infectocontagiosas, em que a carência de tratamento adequado faz com que enfermos permaneçam transmissores incluso ${ }^{23}$. Além do atraso na busca do tratamento mais adequado, interações entre medicamentos, risco de reações adversas, toxicidade e abuso no consumo de medicamentos tornam-se possíveis.

Apesar de não ser um fenômeno único da modernidade, o consumo de medicamentos sem prescrição tem se tornado uma prática comum na população brasileira em todos os grupos etários $^{24}$. Em 2001, 80 milhões de pessoas praticaram a automedicação, e cerca de 20 mil morrem ao ano em sua decorrência ${ }^{25}$. 0 incumprimento generalizado das normas de comercial ização dos medicamentos sujeitos à prescrição e as estruturas públicas de saúde que não absorvem a demanda sobre o setor tornam a prática da automedicação uma opção ao refletir as carências e hábitos culturais de nossa sociedade. As demandas por atenção à saúde parecem ser reforçadas pelas estratégias de promoção e publicidade de 
medicamentos veiculadas à população e aos responsáveis pelas vendas no varejo. A sociedade brasileira se encontra excessivamente exposta à propaganda de medicamentos ${ }^{26}$, sem ter 0 devido esclarecimento sobre os riscos associados ao seu uso. Ademais, a forma de remuneração dos atendentes das farmácias e drogarias brasileiras, baseada em comissão sobre vendas, cria uma lógica de mercado que favorece a prática da automedicação.

Diante do exposto, esse trabalho se propôs a investigar a ocorrência da automedicação e realizar uma avaliação de risco desta prática em um grupo de idosas do Distrito Federal, por meio de pesquisa realizada em ambulatório hospitalar universitário.

\section{Material emétodos}

Desenvolveu-se um estudo transversal descritivo, mediante entrevista semi-estruturada, com uma amostra de idosas residentes em comunidade no Distrito Federal com idade maior ou igual a 60 anos. Cada idosa foi atendida no ambulatório de Atenção ao Idoso do Hospital da Universidade Católica de Brasília (HUCB) em consulta farmacêutica que permitia o relato de consumo esporádico e/ou induzido por indivíduo não habilitado de produtos com fins terapêuticos. No que se refere às variáveis socioeconômicas das idosas que praticaram automedicação, foram analisadas as condições de escolaridade e de renda familiar mensal.

Foi considerado automedicação o consumo declarado por cada idosa de medicamentos: i) sem prescrição por profissional habilitado, ii) indicados por leigos ou vendedores em farmácias edrogarias, e/ou iii) reaproveitados de terapias anteriores ou preparados de forma caseira (chás, pós, infusões, etc).

Para análise da farmacoterapia e dos PRM s envolvendo a automedicação, foram considerados apenas os medicamentos alopáticos consumidos de forma contínua ao longo dos trinta dias que antecederam a entrevista. Estes medicamentos tiveram seus princípios ativos classificados conforme a lista de medicamentos essenciais da Organização Mundial de Saúde ${ }^{27}$ e a Relação $\mathrm{N}$ acional de M edicamentos Essenciai ${ }^{28}$, respeitando-se a classificação dos fármacos segundo 0 Dicionário Anatômico-Terapêutico-Químico ${ }^{29}$. Para a análise de possíveis interações medicamentosas, analisou-se cada princípio ativo constituinte do grupo dos medicamentos utilizados por autoterapia quanto a sua possibilidade de interação com os demais princípios do mesmo grupo ecom os princípios ativos dos medicamentos prescritos. Para tanto, utilizou-se da lista deinterações potencialmente severas da Organização Mundial da Saúde (OM S) ${ }^{27}$.

Para verificar se a obtenção dos medicamentos ocorreu conforme a regulamentação brasileira de ven $\mathrm{da}^{30}$, cada item foi classificado como: a) produto de venda livre (OTC - over the counter), ou b) produto de venda mediante apresentação de receituário, com ou sem a obrigatoriedade de retenção do mesmo.

0 risco potencial de uso de determinados medicamentos por idosos foi analisado conforme os critérios de Beers ecolaboradores ${ }^{31}$, os quais estabelecem uma relação de medicamentos impróprios para consumo por falta de comprovação de eficácia terapêutica ou por elevação da razão risco/ben efício em seu uso por idosos. Para o presente trabalho, o consumo desse grupo de medicamentos foi investigado utilizando-se da atualização realizada por Fick e colaboradore ${ }^{32}$.

Todos os resultados descritos são referentes aos medicamentos utilizados por autoterapia. A análise estatística realizada consistiu na análise de variância AN OVA ou no teste não paramétrico de qui-quadrado, empregando-se o software SPSS 10 com interval os de confiança de $95 \%$.

\section{Resultados ediscussão}

Foram atendidas 218 idosas em consulta farmacêutica, das quais $77,5 \%(n=169)$ relataram usar algum tipo de medicamento. 0 fato de uma elevada proporção de idosas utilizar algum tipo de medicamento pode ser considerado lugar-comum na literatura científica ${ }^{5,33,34}$, pois se sabeque o uso de medicamentos se acentua desde a quarta década da vida ${ }^{35}$. Das usuárias, 30,8\% ( $\left.n=52\right)$ faziam uso de um ou mais produtos sem prescrição médica, perfazendo 85 eventos de automedicação. Esta freqüência de utilização de medicamentos por automedicação foi maior que a relatada por M iralles ${ }^{36}$, em que $18 \%$ dos idosos usavam produtos adquiridos sem prescrição.

Entre as praticantes de automedicação, a maioria apresentava baixa escolaridade, o que pode ser exemplificado pela freqüência conjunta de indivíduos que não possuíam educação formal $(9,6 \% ; n=5)$ e que relataram ensino fundamental incompleto $(55,7 \% ; n=29)$. Tal conjunto foi responsável por consumir $67 \%(n=57)$ dos medicamentos utilizados em automedicação. Com rela- 
ção à renda familiar mensal, 32,7\% ( $n=17)$ apresentaram renda menor ou igual a um salário mínimo (aproximadamenteU $\$ 143,00$ à época), sendo responsáveis por utilizar 32,9\% $(n=28)$ das automedicações. Análises devariância demonstraram que o consumo de medicamentos sem prescrição não se mostrou variar quantitativamente conforme a renda e a escolaridade das pacientes, indicando que essa prática ocorre de forma homogênea no segmento geronte da população.

No presente estudo, os medicamentos alopáticos representaram a maioria $(86 \% ; n=73$ ) dos produtos envolvidos com automedicação, compreendendo o principal grupo em estudo, uma vez que medicamentos caseiros e fitoterápicos constituíram 9\% $(n=7)$ do total de medicamentos usados em autotratamento pelas idosas. A classe demedicamentos com maior freqüência de utilização por automedicação consistiu no grupo dos analgésicos, antipiréticos e antiinflamatórios $(44,7 \% ; n=38)$, sucedido pel os grupos dos medicamentos para o trato gastrintestinal, suplementos minerais e vitamínicos, medicamentos para 0 sistema cardiovascular e antialérgicos que, em conjunto, representaram $30,7 \%(n=26)$ do total (Tabela 1). 0 cenário exposto revela que o perfil de medicamentos associados com automedicação assemelha-se ao perfil de classes terapêuticas mais utilizadas pelo segmento idoso da população brasileira2 ${ }^{22,37,38}$. Testes estatísticos não paramétricos fal haram em demonstrar que o consumo das classes terapêuticas utilizadas em automedicação variava por influência do consumo de outra classe de produtos, prescritos ou automedicados. Pelo exposto, os resultados sugerem que a prática de automedicação no país se dá em resposta ao perfil de morbidades instaladas na população brasileira, em detrimento da concepção de que o idoso recorre ao autocuidado para a prevenção de morbidades ou para dirimir males designificância clínica pouco rel evantes.

Um achado que corrobora a argumentação exposta acima deriva da análise da automedicação quanto à forma de obtenção. Apesar de a maioria $(52,9 \% ; n=45)$ do total demedicamentos envolvidos com automedicação constituírem artigos que dispensavam apresentação de receituário, observou-se que um em cada três desses produtos exigia apresentação de prescrição mé dica para obtenção segundo a legislação brasilei$\mathrm{ra}^{30}$. Diclofenaco e ranitidina, exemplos de fármacos de venda sob prescrição, podiam ser encontrados na composição de $10,6 \%(n=9)$ e $4,7 \%(n=4)$ desses medicamentos, também tendo sido encontrados por M osegui e colaboradores $^{6}$ e por Nóbrega e colaboradores ${ }^{38}$ entre os princípios ativos mais utilizados por idosos brasileiros que residem em comunidade.

$\mathrm{Na}$ análise das interações medicamentosas envolvendo princípios ativos sem prescrição, foram encontrados dez diferentes eventos de interações potencialmente danosas para a saúde (Tabela 2). Em nossas condições, nifedipino correspondeu ao princípio ativo mais freqüentemente encontrado em eventos de interação medicamentosa $(n=4)$, seguido pelo diclofenaco $(n=3)$ e por hidroclorotiazida $(n=3)$. Estes achados revelam que medicamentos cardiovasculares e antiinflamatórios corresponderam à maior parcela dos princípios ativos associados à automedi-

Tabela 1. Classes terapêuticas de maior freqüência na prática de automedicação avaliada.

\begin{tabular}{lccc}
\hline \multicolumn{1}{c}{ Classes terapêuticas } & $\begin{array}{c}\text { Eventos } \\
(\mathrm{n})\end{array}$ & $\begin{array}{c}\text { Freqüência } \\
\text { relativa(\%) }\end{array}$ & $\begin{array}{c}\text { Freqüência } \\
\text { cumulativa(\%) }\end{array}$ \\
\hline Analgésicos, antipiréticos e antiinflamatórios & 38 & 44,7 & 44,7 \\
M edicamento para o trato gastrintestinal & 9 & 10,6 & 55,3 \\
Suplementos minerais e vitamínicos & 6 & 7,1 & 62,4 \\
Cardiovasculares & 6 & 7,1 & 69,5 \\
Antialérgicos & 5 & 5,9 & 75,4 \\
Outros (alopáticos) & 9 & 10,6 & 86,0 \\
Medicamentos caseiros ou fitoterápicos & 12 & 14,0 & 100,0 \\
Total & 85 & 100,0 & 100,0 \\
\hline
\end{tabular}


Tabela 2. Potenciais interações medicamentosas dos princípios ativos constituintes dos medicamentos de autoterapia com os demais medicamentos de autoterapia ou prescritos.

\begin{tabular}{|c|c|c|}
\hline Princípios ativos & $\begin{array}{l}\text { Severidade } \\
\text { da interação }\end{array}$ & $\begin{array}{l}\text { Justificativa para o grau } \\
\text { de severidade da interação }\end{array}$ \\
\hline Hidroclorotiazida e Diclofenaco & Baixa & $\begin{array}{l}\text { Diclofenaco diminui o efeito diurético da hidroclorotiazida } \\
\text { por bloquear a produção de prostaglandinas. }\end{array}$ \\
\hline Betametasona e Diclofenaco & Baixa & Risco de úlcera ou hemorragia gastrintestinal. \\
\hline Hidroclorotiazida e Enalapril & Alta & Desencadeia efeitos hipotensivos (extremos). \\
\hline Diclofenaco e Paracetamol & Baixa & Paracetamol reduz a biodisponibilidade do diclofenaco \\
\hline M etildopa e Timolol & Alta & $\begin{array}{l}\text { Em alguns casos, o uso combinado destes fármacos pode } \\
\text { aumentar a pressão sanguínea. }\end{array}$ \\
\hline Ranitidina e Paracetamol & Baixa & $\begin{array}{l}\text { Ranitidina inibe a metabolização do paracetamol } \\
\text { aumentando os níveis plasmáticos desse fármaco. }\end{array}$ \\
\hline Fluoxetina e Nifedipino & M oderada & $\begin{array}{l}\text { Fluoxetina bloqueia a degradação do nifedipino pelo } \\
\text { metabolismo hepático. }\end{array}$ \\
\hline Nifedipino e Hidroclorotiazida & Alta & Desencadeia efeitos hipotensivos \\
\hline Nifedipino e Propanolol & Alta & Hipotensão severa e falência cardíaca ocasional \\
\hline Nifedipino e I sosorbida & Alta & Provoca efeitos hipotensivos \\
\hline
\end{tabular}

cação com potencial para desencadear algum PRM. Segundo critérios da OM S27, cinco das interações verificadas podem ser consideradas potencialmente graves independentemente daidade do usuário (Tabela 2). Assim sendo, as formas deinteração medicamentosa encontradas são re levantes para o estado de saúde do idoso, haja vista que idosos normalmente apresentam-se mais susceptíveis aos efeitos terapêuticos e nocivos dos fármacos devido às alterações fisiológicas que acompanham o envelhecimento ${ }^{31,39}$.

Quanto à adequação para a faixa etária, foi observado que parcela dos fármacos utilizados por automedicação pertenciam ao conjunto de medicações cujo consumo por pacientes idosos podeser considerado impróprio. Segundo os critérios mais recentes ${ }^{32}$, nove dos princípios ativos automedicados podem ser considerados potencialmente perigosos para uso por idosos, a saber: diclofenaco $(n=9)$, orfenadrina $(n=4)$, carisoprodol ( $n=3)$, ciclobenzaprina $(n=3)$, nifedipino $(n=2)$, clonidina $(n=1)$, dexclorfeniramina $(n=1)$, piroxican ( $n=1$ ) e metildopa ( $=1$ ). Excetuando-se a clonidina, que apresenta baixa potencialidade de desenvolver reação adversa para o idoso, os demais fármacos apresentam risco de reações adversas com grau de severidade que pode ser classificado como alto. Ape- sar de se saber que o uso desses produtos per se não determina que resultados adversos ocorrerão, o uso desses medicamentos suscita preocupação na medida em que compõem um conjunto de fármacos que deveriam requerer consideração clínica cuidadosa por parte dos prescritores e farmacêuticos ${ }^{31}$. Isso pode ser exemplificado pelo fato de que apenas a orfenadrina constitui medicamento de venda livre, segundo a legislação brasileira.

Em nossas condições, vale ressaltar que não foram encontrados eventos de redundância farmacológica envolvendo os produtos usados por automedicação, quer seja entre os medicamentos desse grupo, quer seja entre os desse grupo e os medicamentos prescritos.

\section{Conclusão}

O estudo apresentado corrobora a noção vigente de que a prática da automedicação constituisefreqüente entre idosos que residem em comunidade, haja vista que um em cada três usuários de medicamentos fazia uso de ao menos uma especiali dade farmacêutica al opática sem indicação por profissional habilitado. Ademais, o consumo desses produtos não se mostrou variar 
conforme renda, grau de escolaridade ou progressão etária. Perfil semelhante foi observado por Villarino e colaboradores ${ }^{40}$, em município do Sul do Brasil, onde as barreiras socioeconômicas assim como a insatisfação com a qualidade do serviço de saúde não semostraram os principais determinantes da automedicação.

0 presente estudo revela ainda como a prática da automedicação pode constituir fator de risco para problemas relacionados a medicamentos. Conforme observado, a utilização de medicamentos sem indicação por profissional habilitado pode aumentar o número de substâncias usadas por idosas, levando ao mascaramento desintomas, a interações medicamentosas e a efeitos adversos possíveis. A comercialização sem receita de medicamentos que deveriam ser vendidos apenas sob prescrição contribuiu para o cenário de autoterapia detectada, pois parcela expressiva ( $n=28 ; 32,9 \%$ ) dos eventos de medicação nãoassistida envolviam produtos que exigiam apresentação de receituário segundo a legislação vigente. A adoção da prática de retenção de receita dos medicamentos de venda sob prescrição poderia ser uma medida eficaz de coibir a autome- dicação por produtos que deveriam ser usados com supervisão.

Pelo exposto, o quadro geral desaúdedo idoso brasileiro poderia ser alvo demelhoramentos pelo cumprimento rigoroso da legislação em saúde e pela implementação de medidas eficientes de atenção farmacêutica, tanto no sistema público quanto no sistema privado de saúde, para a promoção da saúde e para a qualidade de vida do idoso. A comercialização e consumo, sem qual quer tipo de barreira, de medicamentos sujeitos a prescrição e que envolvem risco à saúde sugerem a necessidade de regulamentação e fiscalização mais rigorosas da comercialização e dispensação de medicamentos no Brasil.

Finalmente, pode-se acrescentar que o medicamento ocupa papel central na busca pela recuperação da saúde e elemento essencial das práticas profissionais. A disponibilidade desses produtos pode satisfazer as expectativas dos usuáriOS, mas deve ser considerada pelos profissionais de saúde como uma ferramenta adicional, acessória às medidas de caráter preventivo e de promoção da saúde da população, sobretudo no tocante ao segmento idoso. 


\section{Colaboradores}

PC Bortolon trabal hou na concepção do estudo e seu delineamento, na consecução da metodologia, na análise e interpretação dos resultados e na redação do manuscrito; EFF M edeiros trabaIhou na concepção da pesquisa e na consecu ção da metodologia; JOS Naves trabalhou na revisão crítica para aprovação do artigo; M GO Karnikowski trabalhou na concepção do estudo e seu delineamento, na análise e interpretação dos resultados ena redação do manuscrito eOT Nóbrega trabalhou no delineamento do estudo, na coordenação da pesquisa, na análise e interpretação dos resultados, na redação do manuscrito e na revisão crítica para aprovação do artigo.

\section{Agradecimentos}

Este trabal ho foi realizado com recursos da Universidade Católica de Braślia (SI GEP - edital 01/ 2005).

\section{Referências}

1. Chaimowicz F. A saúde dos idosos brasileiros às vésperas do século XXI: problemas, projeções e alternativas. Rev. Saúde Pública 1997; 31(2):184-200.

2. Gordilho A, Nascimento JS, Silvestre J, Ramos LR, Freire MPA, Espindola N, Maia R, Veras R, Karsch $U$. Desafios a serem enfrentados no terceiro milênio pelo setor saúde na atenção integral ao idoso. Rio de Janeiro: Universidade Aberta da Terceira Idade/ Universidade do Estado do Rio de Janeiro; 2000.

3. Camarano AA, Kanso S, Pasinato MT, M ello JLE. Idosos brasileiros - indicadores de condições de vida e de acompanhamento de políticas. Brasília: Presidência da República/Subsecretária de Recursos Humanos; 2005.

4. Veras RP. Em busca de uma assistência adequada à saúde do idoso: revisão da literatura e aplicação de um instrumento de detecção precoce e de previsibilidade de agravos. Cad Saúde Pública 2003; 19(3):705-715.

5. Rosenfeld $\mathrm{S}$. Prevalência, fatores associados e mau uso de medicamentos entre os idosos: uma revisão. Cad Saúde Pública 2003; 19(3):717-724.

6. M osegui GBG, Rozenfeld S, Veras RPV, Vianna CM M . Avaliação da qualidade do uso de medicamentos em idosos. Rev. Saúde Pública 1999; 33(5):437-444.

7. Hobson M. Medication in older patients. West J M ed 1992; 157(5):539-543.

8. Daly MP, Lamy PP, Richardson JP. Avoid polypharmacy and iatrogenesis in the nursing home. $\mathrm{Md}$ M ed J 1994; 43(2):139-144.

9. Castellar JI, Karnikowski M GO, Vianna LG, Nóbrega OT. Estudo da farmacoterapia prescrita a idosos em instituição brasileira de longa permanência. Acta Med Port 2007; 20(2):97-105.

10. Laukkanen $P$, Heikkinen $E$, Kauppinen $M$, Kallinen $M$. U se of drugs by non-institutionalized urban Finns born in 1904-1923 and the association of drug use with mood and self-rated health. Age Ageing 1992; 21(5):343-352.

11. Psaty BM. Assessing the use of medications in the elderly: M ethods and initial experience in the cardiovascular health study. J Clin Epidemiol 1992; 45(6):683-692.

12. Chrischilles EA, Lemke JH, Wallace RB, Drube GA. Prevalence and characteristics of multiple analgesic drug use in an elderly study group. J Am Geriatr Soc 1990; 38(9):979-984.

13. Hanlon JT, Schmader KE, Koronkowski MJ, Weinberger M, Landsman PB, Samsa GP, Lewis IK. Adverse drug events in high risk older outpatients. J Am Geriatr Soc 1997; 45(8):945-948.

14. Ivama AM, organizadora. Consenso brasileiro de atenção farmacêutica: proposta. Braślia: Organização Pan-Americana da Saúde; 2002.

15. Perry DP. When medicine hurts instead of helps. Consult Pharm 1999; 14:1326-1330.

16. Loyola Filho AI, Uchoa E, Guerra HL, Firmo JOA, Lima-Costa MF. Estudo de base populacional sobre o consumo de medicamentos entre idosos: Projeto Bambuí. Cad Saúde Pública 2005; 21(2):545-553. 
17. Fundação Oswaldo Cruz. Centro de Informação Científica e Tecnológica. Sistema Nacional de Informações Tóxico-Farmacológicas. Rio de Janeiro: 2006 [acessado 2006 Mai 22]. Disponível em: http://www. fiocruz.br/sinitox/2003/umanalise2003.htm 1998

18. Pfaffenbach G, Carvalho OM, Bergsten-M endes G Reações adversas a medicamentos como determinantes da admissão hospitalar. Rev Assoc M ed Bras 2002; 48(3):237-241

19. Bootman JL, Harrison DL, Cox E. The health care cost of drug-related morbidity and mortality in nursing facilities. Arch Intern M ed 1997; 157(18):2089-2096.

20. Brasil. M inistério da Saúde. Secretária de Políticas de Saúde. Departamento de Atenção Básica. Política nacional de medicamentos. Brasília: Ministério da Saúde; 2001.

21. Naves JOS. Orientação farmacêutica para DST nas farmácias do DF: um estudo de intervenção [tese]. Brasília (DF): Universidade de Brasília; 2006.

22. Castro LLC, Costa AM, Kozoroski AM, Rossini A, Cymrot R. Algumas características da pratica da automedicação em Campo Grande, M ato Grosso do Sul. Saúde Farmacol 2000; 2:4-10.

23. Organização Mundial de Saúde. International Pharmaceutical Federation. The role of the pharmacist in the fight against the HIV-AIDS pandemic. a joint declaration between the WHO and IPF. Genebra: OM S; 1997.

24. Arrais PSD, Coelho HLL, Batista MCDS, Carvalho $M L$, Righi RE, Arnau JM. Perfil da automedicação no Brasil. Rev. Saúde Pública 1997; 31(1):71-77.

25. Instituto Virtual de Fármacos. Rio de Janeiro: 2006 [acessado $2006 \mathrm{Fev}$ 02]. Disponível em: http:// www.ivfrj.ccsdecania.ufrj.br/ivfonline/edicao_0012/ automedicacao.html

26. Automedicação [editorial]. Rev Assoc M ed Bras 2001; 47(4):269-270

27. Organização M undial de Saúde. WHO M odel Formulary. Oslo: OM S; 2004.

28. Brasil. M inistério da Saúde. Departamento de Atenção Básica. Gerência Técnica de Assistência Farmacêutica. Relação $\mathrm{N}$ acional dos M edicamentos Essenciais. Brasília: M inistério da Saúde; 2002.

29. Organização Mundial de Saúde. ATC Index. Oslo: OM S; 2002.
30. Brasil. Agência Nacional de Vigilância Sanitária. Re solução RDC no 138 de 29 de maio de 2003. Dispõe sobre 0 enquadramento na categoria de venda de medicamentos. Diário Oficial da União 2003; 02 jun.

31. Berrs MH. Explicit criteria for determining potentially inappropriate medication use by the elderly. Arch Intern M ed 1997; 157(14):1531-1536.

32. Fick DM, Cooper JW, Wade WE, Waller JL, Maclean JR, Beers M H. U pdating the Beers criteria for potentially inappropriate medication use in older adults: results of a US consensus panel of experts. Arch Intern M ed 2003; 163(22):2716-2724.

33. Coelho Filho JM, M arcopito LF, Castelo A. Perfil de utilização de medicamentos por idosos em área urbana do nordeste do Brasil. Rev. Saúde Pública 2004; 38(4):557-564.

34. Anderson G, Kerluke K. Distribution of prescription drug exposures in the elderly: description and implications. J Clin Epidemiol 1996; 49(8):929-935.

35. Bardel A, Wallander MA, Svardsudd K. Reported current use of prescription drugs and some of its determinants among 35 to 65-year-old women in mid-Sweden: a population-based study. J Clin Epidemiol 2000; 5(6):637-643.

36. M iralles M A. Access to care and medication use among the ambulatory elderly in Rio de Janeiro, Brazil [thesis]. Florida: University of Florida; 1992.

37. Cordeiro H. A indústria da saúde no Brasil. Rio de Janeiro: Graal; 1980.

38. Nóbrega OT, M elo GF, Karnikowski M GO. Pattern of drugs prescribed for community-residing middle-aged and older adults from the outskirts of Brasília. Rev Bras Ciênc Farm 2005; 41(2):271-277.

39. Nóbrega OT, Karnikowski M GO. Pharmacotherapy in the elderly: precautions with medications. Cien Saude Colet 2005; 10(2):309-313.

40. Vilarino JF. Perfil da automedicação em município do sul do Brasil. Rev. Saúde Pública 1998; 32(1):43-49.

Artigo apresentado em 14/08/2006

Aprovado em 30/07/2007

Versão final apresentada em 08/08/2007 